\title{
The effect of XELOX plus bevacizumab on rectal hepatoid adenocarcinoma
}

\author{
Kazunori Otsuka • Hiroshi Nanjo • Hiroshi Soeda • \\ Hiroyuki Shibata
}

Received: 26 May 2012/ Accepted: 21 August 2012/Published online: 25 October 2012

(C) The Japan Society of Clinical Oncology 2012

\begin{abstract}
Colorectal hepatoid adenocarcinoma is rarely reported and is known to have a poor prognosis. Reports of chemotherapy against colorectal hepatoid adenocarcinoma are scarce. Here, we provide the first report of a case involving XELOX + bevacizumab treatment of rectal hepatoid adenocarcinoma. The patient, a 40-year-old female, was diagnosed with early rectal cancer and underwent endoscopic mucosal resection. Fourteen months later, multiple lymph node metastases appeared. She received XELOX + bevacizumab, and maintained stable disease for approximately ten months. However, unfortunately, this regimen had to be stopped because of interstitial pneumonitis. She underwent other chemotherapies and chemoradiotherapies but died approximately two years after the recurrence. Our results indicate that the XEL$\mathrm{OX}+$ bevacizumab regimen may be effective for controlling this disease.
\end{abstract}

Keywords Colorectal hepatoid adenocarcinoma .

Alpha-fetoprotein · XELOX · Bevacizumab

\section{Introduction}

Hepatoid adenocarcinoma (HAC) is a rare extrahepatic neoplasm that is characterized by morphologic phenotypes similar to those of hepatocellular carcinoma (HCC) $[1,2]$.

K. Otsuka $\cdot$ H. Soeda $\cdot$ H. Shibata $(\bowtie)$

Department of Clinical Oncology, Akita University Graduate

School of Medicine, 1-1-1 Hondo, Akita 010-8543, Japan

e-mail: hiroyuki@med.akita-u.ac.jp

H. Nanjo

Department of Clinical Pathology, Akita University Hospital,

1-1-1 Hondo, Akita 010-8543, Japan
The term HAC was coined for a tumor of the stomach by Ishikura et al. [3]. It has mostly been reported to occur in the stomach (63\%), ovary $(10 \%)$, lung (5\%), gallbladder (4\%), pancreas $(4 \%)$, uterus $(4 \%)$, and rarely in the colorectum (2\%) [4]. We searched the PubMed online database for colorectal HAC (CRC-HAC) and found only 12 case reports [5-16]. According to these few reports, this is a rare disease characterized by poor prognosis. Standard regimens for CRC are those usually applied for adenocarcinomas, which are observed in over $90 \%$ cases of CRC [17]. However, there is still controversy regarding the agents that should be applied for CRC-HAC (for example, standard chemotherapies or chemotherapies for HCC, such as sorafenib). It is also reported that regimens for germ cell tumors have been applied for gastric HAC due to its alphafetoprotein (AFP) production. Furthermore, as the number of published CRC-HAC cases is limited, the reported regimens applied for these diseases are somewhat oldfashioned, such as 5-fluorouracil (5-FU) alone (Table 1). One report has described the effect of agents such as oxaliplatin and irinotecan [16]. In this paper, we report the effectiveness of recently approved agents such as capecitabine, S1, bevacizumab, and panitumumab (P-mab).

\section{Case report}

A 40-year-old female presented to a clinic with bloody feces. She underwent a total colonoscopy that revealed a 7-mm-diameter subpedunculated tumor in the rectum (Fig. 1a). The tumor was resected by EMR. The patient's medical history revealed that she was diagnosed with Turner syndrome with mosaicism of 45,X/46,X,del(Xp) in her twenties. Histopathological findings showed that the tumor consisted of areas of both well-differentiated tubular 
Table 1 Therapeutic outcomes of CRC-HAC in the literature

\begin{tabular}{|c|c|c|c|c|c|c|c|c|c|}
\hline Author & Year & Age & Gender & Primary & Metastasis & Histology & $\begin{array}{l}\text { AFP } \\
(\mathrm{ng} / \mathrm{ml})\end{array}$ & Treatment & Survival (M) \\
\hline Nakajima & 1985 & 50 & M & $\mathrm{R}$ & Liver & $\begin{array}{l}\text { Ad (por, mod), } \\
\text { SCC }\end{array}$ & 3018 & Surg, HAI & $6(\mathrm{~S})$ \\
\hline Sato & 1994 & 43 & M & $\mathrm{R}$ & Liver & Ad (mod) & 941 & Surg, HAI, Cx (5-FU), IFN & $3(\mathrm{~S})$ \\
\hline Taguchi & 1997 & 71 & M & $\mathrm{R}$ & Liver & $\begin{array}{l}\text { Ad (well), } \\
\text { medullary }\end{array}$ & 220000 & Surg, HAE & $12(\mathrm{~S})$ \\
\hline Yachida & 2003 & 59 & M & $\mathrm{T}$ & Liver & Ad (pap, tub) & 12873 & Surg, Cx (5-FU, LV) & $2(\mathrm{~S})$ \\
\hline $\mathrm{Fu}$ & 2006 & 71 & M & $\mathrm{Ce}$ & $\mathrm{LN}$ & Ad (por, mod) & 319 & Surg, Ad-Cx & $\begin{array}{l}60<(\text { alive }) \\
\quad(\mathrm{S})\end{array}$ \\
\hline Borgonovo & 2008 & 42 & M & $\mathrm{R}$ & Liver, LN & Hepatoid & 32000 & Surg, HAE & $18(\mathrm{~S})$ \\
\hline Cappetta & 2012 & 75 & $\mathrm{~F}$ & A & $\begin{array}{l}\text { LN, (PC, } \\
\text { PLC) }\end{array}$ & Ad (por) & 9 & Surg, Cx (FOLFOX, FOLFIRI) & $8(\mathrm{~S})$ \\
\hline Otsuka & & 40 & $\mathrm{~F}$ & $\mathrm{R}$ & $\begin{array}{l}\text { LN, (PLC, } \\
\text { lung) }\end{array}$ & $\begin{array}{l}\text { Ad (well) } \\
\text { Hepatoid }\end{array}$ & 1194 & $\begin{array}{l}\text { EMR, Cx (Xelox + BV, S1, IRI, } \\
\text { P-mab }\end{array}$ & $24(\mathrm{Re})$ \\
\hline
\end{tabular}

Metastatic sites in parentheses were found at fur advanced stage

$R$ rectum, $T$ transverse colon, $C e$ cecum, $A$ ascending colon, $L N$ lymph node, por poorly differentiated, mod moderately differentiated, pap papillary, $A d$ adenocarcinoma, Surg surgical, $H A I$ hepatic arterial infusion, $H A E$ hepatic arterial embolization, $C x$ chemotherapy, $L V$ leucovorin, $(S)$ after surgical treatment, $(R e)$ after recurrence, $P C$ peritonitis carcinomatosa, $P L C$ pleuritis carcinomatosa

adenocarcinoma and poorly differentiated adenocarcinoma (Fig. 1b). The cancer had invaded partly into the superficial layer of the submucosa. The depth of invasion was classified as submucosal invasion (SM1) and the margin was tumor-free. Fourteen months later, a follow-up computed tomography (CT) scan showed multiple swollen lymph nodes from the periaortic to the presacral regions (Fig. 2a, b), and the patient was subsequently admitted to our hospital. ${ }^{18}$ Fluorodeoxyglucose-positron emission tomography $\left({ }^{18}\right.$ FDG-PET) revealed an abnormally increased uptake in multiple lymph nodes, including those in not only periaortic and presacral regions but also the left subclavicular and paraesophageal regions (Fig. 2c, d). As the tumors were possibly a cancer of unknown primary, various serum tumor markers were examined, and she showed elevated serum levels of AFP, carcinoembryonic antigen (CEA), and carbohydrate antigen 19-9 (CA19-9). The levels of the serum AFP, CEA, and CA19-9 were $1194.2 \mathrm{ng} / \mathrm{ml}$, $48.1 \mathrm{ng} / \mathrm{ml}$, and $38.9 \mathrm{U} / \mathrm{ml}$, respectively. Because of the unusually elevated AFP levels, we performed an immunohistochemical examination of the archival EMR specimen. The tumor was composed of two different types of cancer cells: one type was well-differentiated tubular adenocarcinoma, while the other was poorly differentiated solid adenocarcinomas with slightly eosinophilic cytoplasm and enlarged nuclei, characteristic of hepatoid adenocarcinomas. The poorly differentiated adenocarcinomas were AFP-positive. These cells were considered to be possible hepatoid adenocarcinomas (Fig. 1c). As a result, we diagnosed multiple lymph node metastasis derived from the resected AFP-producing rectal cancer. The detailed diagnostic process in this case has already been reported elsewhere in Japanese. Although there are no established regimens for $\mathrm{CRC}-\mathrm{HAC}$, based on the previous reports, the patient was treated with chemotherapy with an oxaliplatin plus capecitabine $(\mathrm{XELOX})+$ bevacizumab $(\mathrm{BV})$ regimen (oxaliplatin, $130 \mathrm{mg} / \mathrm{m}^{2}$ on day 1; capecitabine, $2000 \mathrm{mg}$ / $\mathrm{m}^{2}$ on days $1-14 ; \mathrm{BV}, 7.5 \mathrm{mg} / \mathrm{kg}$ on day 1 , every three weeks) for up to 14 courses. Several CT examinations over the next ten months showed that the metastatic lymph nodes were approximately the same size, and that no new lesions had appeared. This patient was categorized as having stable disease (SD) according to the Response Evaluation Criteria in Solid Tumors (RECIST) criteria (version 1.1) (Fig. 2e, f). However, the patient developed grade 2 interstitial pneumonitis, as defined by the Common Terminology Criteria for Adverse Events (CTCAE) version 4.0 scale. In lieu of these findings, we discontinued the chemotherapy regimen, although other adverse events such as hypertension (grade 2), nausea (grade 2), and urticaria (grade 2) were tolerable. The patient's treatment regime was changed to concurrent chemoradiotherapy (CRT) with S-1 (tegafur, gimeracil, and oteracil potassium, $50 \mathrm{~Gy} / 25$ fractions for periaortic lymph node metastasis), followed by single therapies with $\mathrm{S}-1$ and irinotecan (IRI), one after the other. Furthermore, P-mab therapy was initiated, because the tumor cells contained wild-type codons 12 and 13 of the KRAS gene. However, the disease was not controlled by any treatment other than XELOX $+\mathrm{BV}$ or CRT with S-1. The patient's serum AFP level decreased below $500 \mathrm{ng} / \mathrm{ml}$ during XELOX + BV or CRT with S-1 therapies. Similarly, CEA and CA19-9 decreased to normal levels (20.7 and $13.7 \mathrm{ng} / \mathrm{ml}$, respectively) with these treatments. The second treatment with CRT (to the 

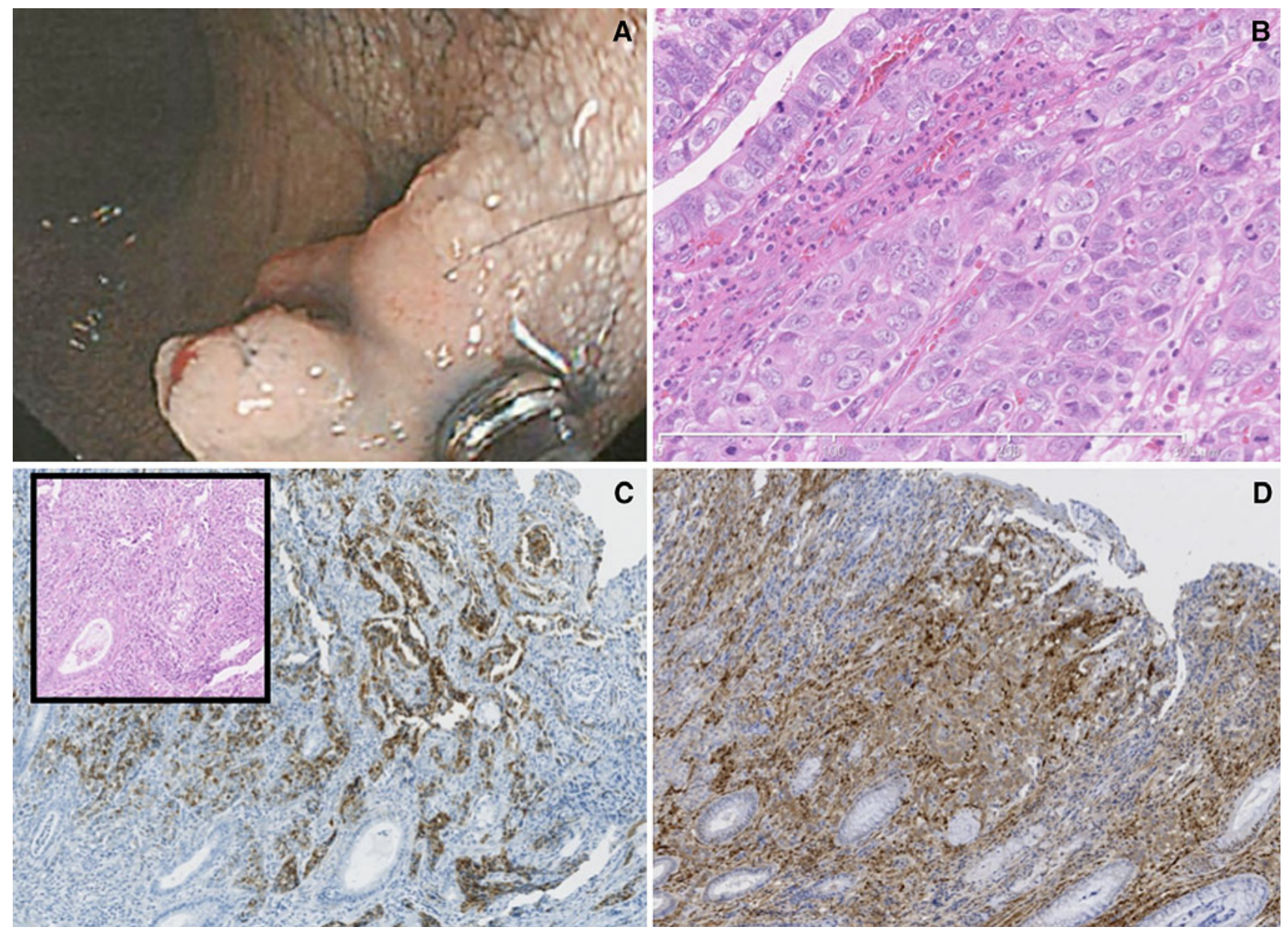

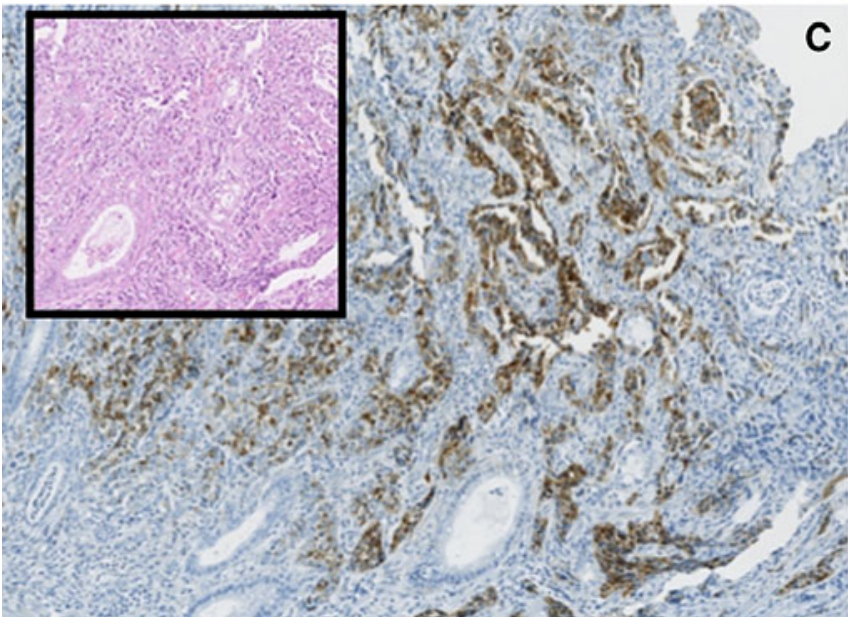

Fig. 1 Clinicopathological features of the rectal tumor. a Endoscopic view. b Histopathological findings for the EMR specimen. The tumor consisted of both well-differentiated tubular adenocarcinomas and poorly differentiated solid adenocarcinomas with slightly eosinophilic cytoplasm and enlarged nuclei, characteristic of hepatoid

presacral regions and the left subclavicular lymph nodes) and S-1 also decreased the serum AFP level; however, the response remained within the SD criteria. The other treatments did not decrease the serum AFP level any further, or the CEA and CA19-9 levels (Fig. 3). The patient had bulky lymph node metastases but no blood-borne metastases for 21 months after her relapse. Twenty-four months after the recurrence, the patient died of multiple lung metastases.

\section{Discussion}

Colorectal hepatoid adenocarcinoma is rare, and reports of chemotherapy for its advanced stage are limited. Only seven studies describing chemotherapy have been reported (Table 1), and four of them described hepatic arterial infusion or embolization against liver metastasis, as most of the reported cases showed hematogenous spread. adenocarcinomas. c Immunohistochemical staining of the rectal tumor for AFP. Immunohistochemical analysis showed that the poorly differentiated adenocarcinomas were AFP-positive. The inset indicates H\&E staining of the same lesion. d Immunohistochemical staining for thymidine phosphorylase

Lymphogenous spread is not typical [14], but it has been mentioned more often in recent publications than in those published previously. Around 2003, 5-fluorouracil (5-FU) was used in systemic chemotherapy of CRC-HAC. A recent publication described the combined therapy of 5-FU with oxaliplatin or IRI, which is standard for general CRC, in which a lower survival rate (eight months) was achieved than the usual median survival time (MST) of CRC. It is still unclear whether CRC-HAC is resistant to the standard regimens for CRC. It was reported that sorafenib, approved for advanced HCC, was used in one case of AFP-producing adenocarcinoma in the peritoneal cavity-an HAC - in which the patient survived for six months [18]. It was also reported that regimens similar to those used for germ-cell tumors were applied in two gastric HAC cases [19]. The treatments were rather effective, but relatively toxic, as shown by the therapy-related death in one case. There are no established regimens for advanced HAC of digestive 

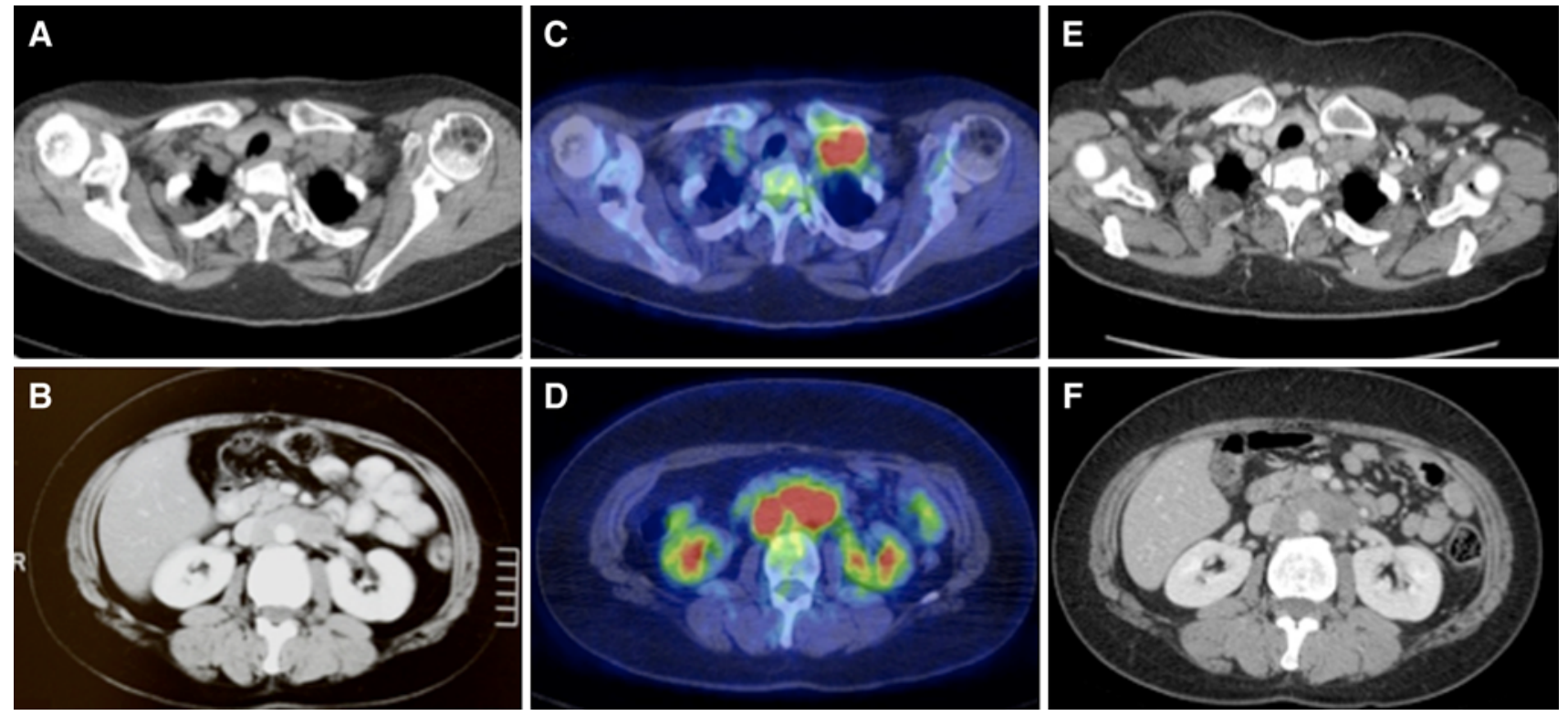

Fig. 2 Imaging of the lymph node metastases. a CT imaging of the subclavian lymph nodes before treatment. b CT imaging of the bulky paraaortic lymph nodes, same as above. c ${ }^{18}$ FDG uptake in the subclavian lymph nodes before treatment. d ${ }^{18}$ FDG uptake in the

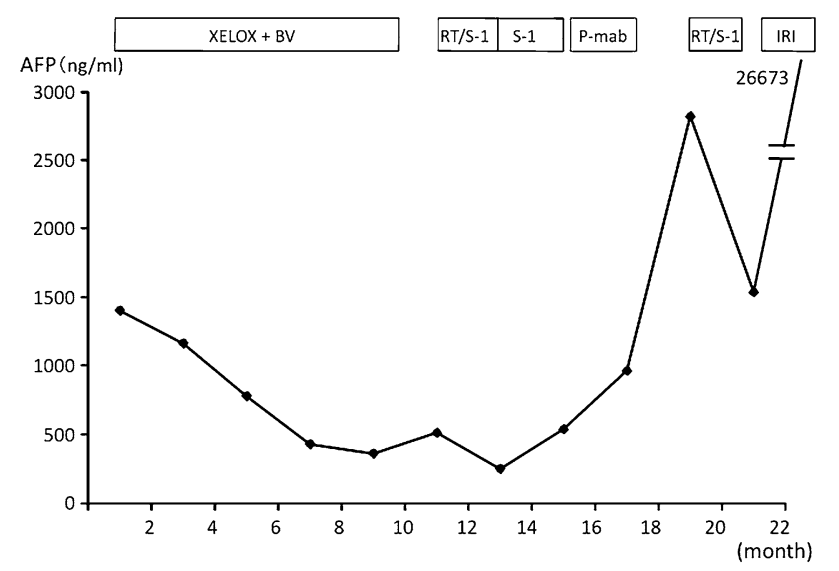

Fig. 3 Change in AFP serum level during treatment. Months after recurrence are indicated

organs except for HCC. In the present report, current standard regimens for advanced CRC, including two molecular-targeted antibodies, were applied. Survival time after recurrence was 24 months, which was comparable to the MSTs associated with typical CRC therapies. Individually, XEL$\mathrm{OX}+\mathrm{BV}$ and CRT with S1 were effective, but IRI and $\mathrm{P}$-mab were not. Thymidine phosphorylase (TP) is upregulated in a wide variety of solid tumors, including colorectal cancer, and it stimulates tumor growth and is associated with poor prognoses [20]. TP is indispensable for the activation of capecitabine, which is converted to 5-FU in the tumor [18]. Indeed, there are some reports which have indicated that high bulky paraaortic lymph nodes, same as above. e CT imaging of the subclavian lymph nodes after five cycles of XELOX + BV treatment. f CT imaging of the bulky paraaortic lymph nodes, same as above

TP expression levels are associated with the response of the tumor to capecitabine [21, 22]. TP expression was confirmed as it was shown experimentally that TP expression was suppressed in an AFP-producing adenocarcinoma cell line [23]. In the present case, immunohistochemical staining for $\mathrm{TP}$ was positive in the initial tumor (Fig. 1d). Actually, this tumor was relatively sensitive to the XELOX regimen. This case indicated that standard CRC regimens can control advanced CRC-HAC. This patient also suffered from Turner syndrome. In a study of cancer incidence in a cohort of 597 women with Turner syndrome, the relative risk of CRC was rather high (6.9) [24]. However, the relationship between CRC-HAC and Turner syndrome is unclear, and there are no reports on CRC-HAC combined with Turner syndrome in the literature.

Acknowledgments The authors would like to thank Enago for the English language review.

Conflict of interest The authors declare that they have no conflict of interest.

\section{References}

1. Ishikura H, Fukasawa Y, Ogasawara K et al (1997) Gastrointestinal hepatoid adenocarcinoma: venous permeation and mimicry of hepatocellular carcinoma, a report of four cases. Histopathology 31:47-54

2. Glatz K, Terraccino LM, Mhawech P et al (2003) Hepatoid adenocarcinoma with liver metastasis mimicking hepatocellular 
carcinoma: an immunohistochemical and molecular study of eight cases. Am J Surg Pathol 27:1302-1312

3. Ishikura H, Fukasawa Y, Ogasawara K et al (1985) An AFPproducing gastric carcinoma with features of hepatic differentiation. A case report. Cancer 56:840-848

4. Metzgeroth G, Strobel P, Baumbusch T et al (2010) Hepatoid adenocarcinoma-review of the literature illustrated by a rare case originating in the peritoneal cavity. Onkologie 33:263-269

5. Nakajima T, Okazaki N, Morinaga S et al (1985) A case of alphafetoprotein-producing rectal carcinoma. Jpn J Clin Oncol 15:679-685

6. Gonsoulin W, Mason B, Carpenter RJ Jr (1990) Colon cancer in pregnancy with elevated maternal serum alpha-fetoprotein level at presentation. Am J Obstet Gynecol 163:1172-1173

7. Yu YY, Ogino T, Okada S (1992) An alpha-fetoprotein-producing carcinoma of the rectum. Acta Pathol Jpn 42:684-687

8. Sato Y, Sekine T, Ohwada S (1994) Alpha-fetoprotein-producing rectal cancer: calculated tumor marker doubling time. J Surg Oncol 55:265-268

9. Hocking GR, Shembrey M, Hay D et al (1995) Alpha-fetoprotein-producing adenocarcinoma of the sigmoid colon with possible hepatoid differentiation. Pathology 27:277-279

10. Kato K, Matsuda M, Ingu A et al (1996) Colon cancer with a high serum alpha-fetoprotein level. Am J Gastroenterol 91:1045-1046

11. Taguchi J, Yano H, Sueda J et al (1997) Alpha-fetoprotein-producing rectal carcinoma-a case report. Kurume Med J 44:339-348

12. Kurihara K, Konishi F, Kanazawa K et al (1997) Alpha-fetoprotein-producing carcinoma of the colon: report of a case. Surg Today 27:453-456

13. Yachida S, Fukushima N, Nakanishi Y et al (2003) Alpha-fetoprotein-producing carcinoma of the colon: report of a case and review of the literature. Dis Colon Rectum 46:826-831

14. Fu K, Kobayashi A, Saito N et al (2006) Alpha-fetoprotein-producing colon cancer with atypical bulky lymph node metastasis. World J Gastroenterol 12:7715-7716
15. Borgonovo G, Razzetta F, Assalino M et al (2008) Rectal hepatoid carcinoma with liver metastases in a patient affected by ulcerative colitis. Hepatobiliary Pancreat Dis Int 7:539-543

16. Cappetta A, Bergamo F, Mescoli C et al (2012) Hepatoid adenocarcinoma of the colon: what should we target? Pathol Oncol Res 18:93-96

17. Boyle P, Levin B (eds) (2008) World cancer report. IARC, Lyon

18. Eisenhauer EA, Therasse P, Bogaerts J et al (2009) New response evaluation criteria in solid tumours: revised RECIST guideline (version 1.1). Eur J Cancer 45:228-247

19. Germann N, Gross-Goupil M, Wasserman E et al (2001) The chemotherapy of metastatic gastric adenocarcinomas with hypersecretion of alpha-fetoprotein or beta-human chorionic gonadotrophin: report of two cases. Ann Oncol 13:632-636

20. Bronckaers A, Gago F, Balzarini J et al (2009) The dual role of thymidine phosphorylase in cancer development and chemotherapy. Med Res Rev 29:903-953

21. Meropol NJ, Gold PJ, Diasio RB et al (2006) Thymidine phosphorylase expression is associated with response to capecitabine plus irinotecan in patients with metastatic colorectal cancer. J Clin Oncol 24:4069-4077

22. Petrioli R, Bargagli G, Lazzi S et al (2010) Thymidine phosphorylase expression in metastatic sites is predictive for response in patients with colorectal cancer treated with continuous oral capecitabine and biweekly oxaliplatin. Anticancer Drugs 21:313-319

23. Kamoshida S, Suzuki M, Sakurai Y et al (2006) Expression of chemoresistance-related proteins in alpha-fetoprotein-producing adenocarcinoma of the digestive organs. Oncol Rep 16:721-727

24. Hasle H, Olsen JH, Nielsen J et al (1996) Occurrence of cancer in women with Turner syndrome. Br J Cancer 73:1156-1159 